\title{
TODO LO QUE NOS RODEA: ESTUDIO DE REPRESENTACIONES SOCIALES DE LO AMBIENTAL Y LA NATURALEZA EN UNA FACULTAD DE CIENCIAS NATURALES
}

\author{
Everything around us: a study of social representations of both environment \\ and nature in a College of Natural Sciences
}

\author{
Iván Paolocá* \\ https://orcid.org/0000-0002-1503-5914
}

\section{Resumen}

En el presente trabajo analizo las representaciones sociales de lo ambiental y la naturaleza de los estudiantes avanzados y graduados recientes de la Facultad de Ciencias Naturales y Museo de la Universidad Nacional de La Plata (FCNyM-UNLP), en función de ser una de las pocas facultades que comprende dentro de sus carreras a las Ciencias Naturales y las Sociales, al tener las licenciaturas en Antropología, Biología (con cuatro orientaciones) y Geología. Apliqué técnicas cualitativas de entrevistas semi-estructuradas y utilicé los conceptos de antropocentrismo, ecocentrismo y biocentrismo como categorías analíticas. De los entrevistados, el $63 \%$ presentó una perspectiva biocentrista; la visión hegemónica resultó ser ecocentrista (antropocentrista en geología), siendo "todo lo que nos rodea" la definición de ambiente más repetida. Concluyo que para formar profesionales con una visión biocentrista es importante una perspectiva crítica, que conjugue las Ciencias Naturales con la historia y la filosofía, y una práctica docente y de extensión que sea interdisciplinaria, política, territorial y de empatía con el otro. Espero que este trabajo sea un aporte para la discusión actual en torno a la renovación de los planes de estudios de las carreras de la FCNyM, y que de esta manera las problemáticas socioambientales sean un eje transversal a la hora de desarrollar los mismos. La formación de sujetos conscientes y críticos es la única manera de superar la crisis ambiental actual.

\section{Abstract}

$<$ Ambiente $><$ Naturaleza $><$ Representaciones Sociales $><$ Biocentrismo $>$

In this paper I analyze the social representations of both environment and nature among senior undergraduate students and recent graduates of the College of Natural Sciences and Museum of the University of La Plata (FCNyM-UNLP), which is one of the few academic units that has Natural and Social Sciences embedded in their careers, including Anthropology, Biology (with four orientations), and Geology. For this analysis, I applieed qualitative techniques of semi-structured interviews and concepts of anthropocentrism, ecocentrism, and biocentrism as analytical categories. Of the total of interviewees, $63 \%$ presented a biocentric perspective; the hegemonic vision turned out to be ecocentric (anthropocentric in geology), therefore dualistic, being "everything that surrounds us" the most repeated definition of environment. To conclude, in order to train professionals in a biocentric vision, a critical perspective is important, which combines the Natural Sciences with History and Philosophy, and teaching and community outreach practices should be interdisciplinary, political, territorial and empathetic. This work is expected to contribute to the ongoing discussion on the renewal of the curricula from the FCNyM careers. In this regard, the socio-environmental problems become a cross cutting pillar at the time of developing them. The academic formation of conscious and critical subjects is the only way to overcome the current environmental crisis.

$$
<\text { Environment }><\text { Nature }><\text { Social Representations }><\text { Biocentrism }>
$$

Recibido: 31/10/2019

Aceptado: 01/02/2020

\footnotetext{
* Licenciado en Antropología y Becario Doctoral (CONICET). Laboratorio de Etnobotánica y Botánica Aplicada (LEBA), Facultad de Ciencias Naturales y Museo, Universidad Nacional de La Plata, Argentina, ivan.antropologia@gmail.com
} 
Paolocá. Todo lo que nos rodea: estudio de representaciones sociales de lo ambiental y la naturaleza en una facultad...

\section{Introducción}

La crisis ambiental que estamos atravesando en la actualidad es, ante todo, una crisis de sociedad, ya que no se trata de una "crisis ecológica, en términos de pérdida y degradación ambiental, sino una crisis más profunda que incluye los principios de la modernidad, (...) encarnado en el eurocentrismo, la racionalidad instrumental, el mecanicismo como paradigma de conocimiento, es decir, la crisis se plantea a nivel civilizatorio" (García, 2009, p.16). De esta manera, se plantea como urgente revisar y renegociar los discursos que están motorizando prácticas que atentan contra un ambiente sano, dinámico y plural. La crisis ambiental no se supera solamente con el aporte de nuevas tecnologías, sino revisando el factor humano de manera preventiva y conciliando campos del conocimiento en pos del bienestar humano. Las concepciones hegemónicas de ambiente, y otras asociadas como naturaleza, recursos naturales, biodiversidad, ecosistema, están claramente insertadas en las tradiciones de conocimiento occidentales. Cualquiera de esas ideas implica un dualismo, donde se separa naturaleza de sociedad, entendiendo que ésta existe en sí misma, desenvolviéndose con sus propios procesos y estructuras, no relacionadas con los humanos (Descola y Palsson, 2001).

En la disciplina antropológica, la relación de los seres humanos con su medio, tiene una importancia central. Todas las relaciones sociales se producen en interacción con un medio físico donde se vive, el cual influye a los sujetos, y por tanto, estos a él, de una manera dialéctica. Sin embargo, no todos los investigadores entienden esta relación de la misma manera. En la tradición antropológica las posturas llamadas adaptacionistas han dominado la escena desde el origen de la disciplina (Milton, 1997; Durand, 2002), como por ejemplo: la antropogeografía de Friedrich Ratzel, que calibraba condiciones culturales partiendo de mapas de información medioambiental; el posibilismo ambiental de Franz Boas y Alfred Kroeber (Boas, 1964; Durand, 2002), dándole un papel regulador al ambiente sobre los rasgos culturales, a los cuales favorece o limita; y ciertas corrientes dentro de la ecología cultural (Ortner, 1984; Descola y Palsson, 2001) quienes poseen una visión puramente adaptativa de las sociedades a su medio, con las estrategias de subsistencia como nexo.

Como alternativa al adaptacionismo, han surgido diversas corrientes dentro de las cuales podemos destacar la antropología ecológica de Roy Rappaport (Ortner, 1984; Mastrángelo, 2009), quien formula analogías entre poblaciones humanas y biológicas, valiéndose de los métodos de estudio de esta última. Por otro lado, la etnoecología de Stephen Tyler y Ward Goodenough (Fowler, 1979) que entienden a la cultura como modelador del ambiente, centrándose en el estudio de modelos conceptuales desarrollados a partir de la estructura discursiva del conocimiento empírico de los sujetos. Sin embargo, ambas posturas se posicionan, al igual que el determinismo ambiental, en una visión dualista de la relación humano-ambiente, o, dicho en otras palabras, humano-naturaleza. En consonancia con dicho análisis, defino al ecocentrismo como un tipo particular de antropocentrismo - al poseer también una visión cartesiana de la naturaleza concibiendo a esta como una máquina y transformándola en objeto (Gudynas, 1999)-, donde se valora a los seres vivos no humanos y los factores abióticos por sobre las personas y los 
procesos culturales, considerando a su vez a los primeros de manera estática y frágil. Esto conlleva una noción de naturaleza prístina, la cual debe ser separada (y reservada) de la sociedad humana para evitar ser dañada y/o modificada. Si bien propongo esta definición, es necesario aclarar que el término ecocentrismo es utilizado desde diversas posturas éticas, teóricas y políticas (Leopold, 1949; Eckersley, 1992; Mastrángelo, 2015). Por otro lado, recupero los conceptos de exhabitante e inhabitante propuestos por Ingold (2012), para analizar cómo se posicionan los estudiantes avanzados y graduados recientes, aquí entrevistados, con respecto a lo ambiental, ya sea de manera externa o como habitantes internos respectivamente.

Propongo también, como solución a esta encrucijada, una perspectiva biocentrista de acuerdo con Gudynas (1999; 2004; 2010) como adecuada en el estudio de problemáticas ambientales, a partir de la cual, el ser humano y su ambiente, se tornen dos recortes de la misma entidad, viendo por tanto, una relación dialéctica y de mutua influencia, donde las fronteras de ambas se encuentren difíciles de determinar. En palabras de Gudynas (2010, p. 54) "el biocentrismo busca romper con el antropocentrismo, pero éste último es entendido en un sentido más amplio, como un modo de ser en el mundo, una cosmovisión que expresa un tipo de relacionalidad que sustenta la dualidad Naturaleza - Sociedad. Las valoraciones se hacen esencialmente de acuerdo al beneficio o ventaja humana, convirtiendo al ambiente en objetos, para instrumentalizarlos y manipularlos". Esta perspectiva, no busca generar una naturaleza intocada, sino que defiende una igualdad biocéntrica, donde todas las entidades tengan igual derecho a vivir y prosperar.

Una postura similar es sostenida por la etnobiología, que emerge como disciplina de referencia para la investigación sobre la relación naturaleza-cultura a partir de su desarrollo y constitución en campo de estudio. Si bien comienza como un emprendimiento interdisciplinario empírico y descriptivo, ha ido evolucionando y modificando su perspectiva. La definición actualmente adoptada por la Sociedad Internacional de Etnobiología es “el estudio científico de las relaciones dinámicas entre humanos, biota y ambiente" (Society of Ethnobiology, s/f, traducción). Los cambios en este tipo de estudio han sido sintetizados por Hunn (2007) en cuatro fases temporales, de las cuales destacan para esta propuesta la tercera fase, la cual se interesó por la integración de conocimientos y prácticas, haciendo hincapié en las consecuencias ecológicas que tiene la aplicación de esos conocimientos, y la cuarta fase que incorpora la preocupación por la ética de las investigaciones interculturales. Más recientemente, una quinta fase fue agregada por Wyndham et al. (2011) quienes sitúan la etnobiología contemporánea en el contexto de la crisis ambiental global, y la ubica en el campo de la interdisciplina y sus aplicaciones en áreas relacionadas con la conservación biocultural, focalizando en "las necesidades de un mundo sometido a un cambio ecológico rápido y cambiantes políticas económicas” (Wyndham et al., 2011, p.124, traducción).

Para realizar el presente trabajo he seleccionado a la Facultad de Ciencias Naturales y Museo de la Universidad Nacional de La Plata (FCNyM-UNLP), en función de la particularidad de ser una de las pocas facultades que comprende dentro de sus 
Paolocá. Todo lo que nos rodea: estudio de representaciones sociales de lo ambiental y la naturaleza en una facultad...

carreras, a las Ciencias Naturales y las Sociales, al tener las licenciaturas en Antropología, Biología y Geología, las cuales comparten numerosas materias en común. En trabajos previos, se ha destacado a dicha institución como una de las más importante formadoras de especialistas ambientales de Argentina (Mengascini et al., 2003; Ottenheimer et al., 2004). Así mismo, se ha distinguido cómo la vivencia en dicha institución influye en el cambio de las representaciones sociales sobre la ciencia y el qué hacer científico (Mengascini et al., 2004). Al mismo tiempo, y desde del Grupo de Didáctica de las Ciencias (UNLP), Cordero et al. (2011), hacen hincapié en la importancia de una perspectiva ambiental crítica, que revise las prácticas pedagógicas y la construcción del conocimiento con el fin de orientarlos a la formación de sociedades sustentables. En consiguiente, analicé las representaciones sociales -maneras de interpretar y pensar la realidad cotidiana, es decir, el conocimiento social (Jodelet, 1984)- del ambiente y la naturaleza de los estudiantes avanzados y graduados recientes de la FCNyM, a fin de comprender qué factores influyen en la conformación de las distintas perspectivas, y cuál es el rol de su trayectoria por la facultad en general, y por sus carreras en particular, en la conformación de las mismas. Busqué comprender las visiones sobre lo ambiental que guían las prácticas de los futuros profesionales. Se espera que los resultados de este trabajo contribuyan a mejorar la formación de los estudiantes afines a problemáticas ambientales, al comprender mejor cómo formar profesionales conscientes y comprometidos con la crisis ambiental que se vive en la sociedad actual.

\section{Metodología}

En el presente trabajo se desarrollaron técnicas cualitativas de entrevista semiestructurada (Martin, 1995); en el Anexo 1 se adjuntan las preguntas guía. Previo a la realización de las mismas, les solicité la firma de un consentimiento informado (ver Anexo 2), de acuerdo a las leyes de ética vigentes (International Society of Ethnobiology, 2008). La selección de los entrevistados la realicé mediante un muestreo de tipo bola de nieve, y la población de estudio consistió en estudiantes avanzados (que estén en condiciones de cursar materias optativas) y graduados recientes (menos de un año desde el egreso) de las distintas carreras de la Facultad de Ciencias Naturales y Museo (UNLP), es decir, las licenciaturas en Antropología, Geología y las cuatro orientaciones de Biología: Botánica, Ecología, Paleontología y Zoología. No tuve en cuenta a los estudiantes de la licenciatura en Geoquímica, debido al escaso número de alumnos regulares de la misma. La incorporación de los entrevistados finalizó en aquel punto de saturación donde no se adicionó información novedosa al registro, dando por resultado un total de veintisiete casos: cuatro de Botánica, cinco de Ecología, cuatro de Zoología, cuatro de Paleontología, cinco de Antropología y cinco de Geología. Posteriormente analicé los resultados mediante la comparación de las representaciones de los sujetos, según propone Da Silva et al. (2014, p. 105) para estudios de representaciones. Los nombres de los entrevistados serán cambiados, con el fin de preservar la identidad de los mismos (se reemplazarán por B1-4 en el caso de los de Botánica, E1-5 en el de Ecología, Z1-4 en el de Zoología, P1-4 en el de Paleontología, A1-5 en el de Antropología y G1-5 
en el de Geología). Las frases escritas entre comillas corresponden a citas textuales recogidas de las entrevistas.

\section{Resultados}

De los entrevistados, 17 presentaron una visión más ligada a la biocentrista (63\% del total), de los cuales resultaron ser cinco de Antropología (100\% de dicha carrera), tres de Paleontología (75\%), tres de Botánica (75\%), dos de Zoología (50\%), dos de Ecología (40\%) y dos de Geología (40\%) (ver tabla 1). Por otro lado, fue llamativo como a la hora de definir lo ambiental en una primera instancia el $52 \%$ (71\% si observamos solo a los de Biología, ver figura 1) dijo "todo lo que nos rodea" (ver figura 2). A continuación se desarrollan los resultados carrera por carrera.

Tabla 1. Disposición de resultados respecto a la perspectiva de los entrevistados de las distintas carreras: Botánica (B), Ecología (E), Zoología (Z), Paleontología (P), Geología (G) y Antropología

(A)

\begin{tabular}{cccccccc} 
& B & E & Z & P & G & A & Total \\
\hline Biocentrista & 3 & 2 & 2 & 3 & 2 & 5 & 17 \\
\hline Ecocentrista & 1 & 3 & 2 & 1 & 2 & 0 & 9 \\
\hline Antropocentrista & 0 & 0 & 0 & 0 & 1 & 0 & 1 \\
\hline Total & 4 & 5 & 4 & 4 & 5 & 5 & 27 \\
\hline
\end{tabular}

Figura 1. Definición de ambiente de los entrevistados de biología según la respuesta haya sido "todo lo que nos rodea" u otra

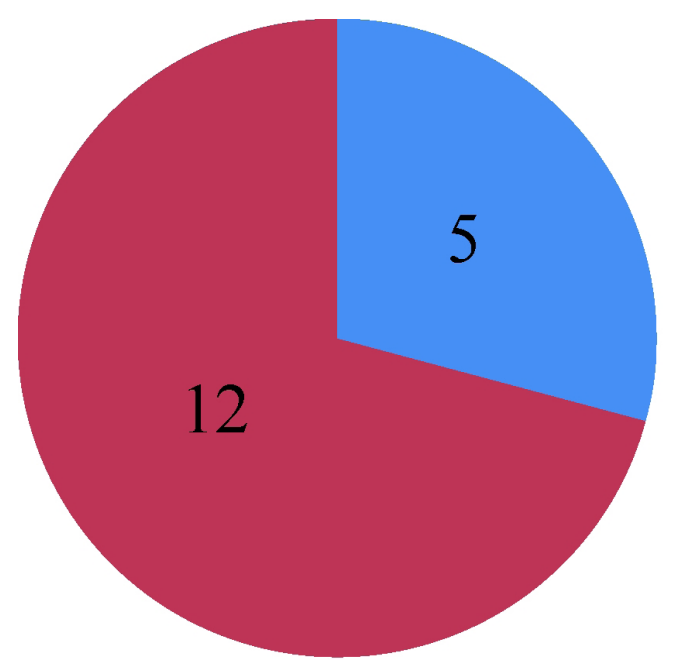

Todo lo que nos rodea

Otras 
Paolocá. Todo lo que nos rodea: estudio de representaciones sociales de lo ambiental y la naturaleza en una facultad...

Figura 2. Diferenciación por carreras de entrevistados que hayan respondido "todo lo que nos rodea" al ser interrogados sobre la definición de ambiente

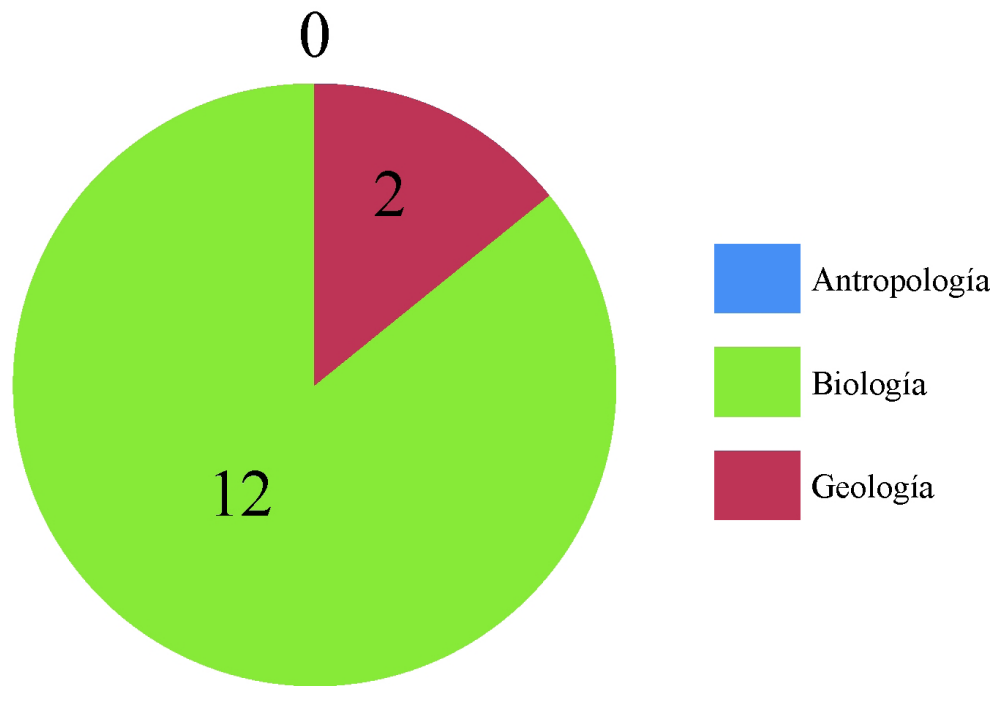

\section{Licenciatura en Biología, orientación Botánica}

Los cuatro entrevistados de la carrera de Botánica, definen al ambiente como "todo lo que nos rodea", sin embargo, al ahondar en el término los resultados fueron disímiles entre sí. Tanto B1, como B3 y B4, se observan a sí mismas como inhabitantes, es decir con influencia sobre su entorno y éste sobre ellos. Siguiendo esta misma línea, sus posturas se acercarían a un biocentrismo. Yendo al detalle, B1 relata cómo antes de trabajar en el CEAMSE, tenía una visión de las personas como "destructoras de la naturaleza" (ecocentrista) la cual era frágil y había que conservarla. Luego de su voluntariado, empezó a entender al basural como un ambiente y fue ahí donde vio las limitaciones de la carrera, pues en la misma le habían enseñado que los ambientes solo estaban compuestos por naturaleza y que los humanos eran parte de ésta. Dice que para tratar problemáticas ambientales "hay que ser burócrata", es decir estar formado en gestión ambiental, lo cual es una formación inexistente en la carrera.

Por su parte, B3 cuenta que ella "hizo un click" en el último año del secundario, al tener un profesor de geografía que les habló de diversos conflictos socioambientales (megaminería y agrotóxicos). Ahí empezó a observar lo ambiental de otra manera, lo cual se profundizó al cursar el primer año de la facultad. Al ver la gran diversidad biológica existente se volvió "soberanista" y se empezó a interesar por realizar prácticas de docencia y extensión, lo cual le enseñó a "no subestimar a la gente". Además, remarca como importante en la conformación de su perspectiva, la interdisciplina, al relacionarse con gente de otras carreras y haber cursado materias más sociales como Antropología General y Botánica Aplicada. Es eso lo que dice que más necesita la facultad: generar "jornadas para encontrarnos", para que las diferentes profesiones trabajen en conjunto. 
En cuanto a su conceptualización de la naturaleza, primero se refiere a ella como lo que no cambia, pero luego dice que sin embargo evoluciona, por lo que finalmente no está muy segura de qué sería, a juzgar por sus propias palabras.

En cuanto a B4, nombra como fundamental el conocer la visión de "los pueblos originarios" sobre la naturaleza y lo ambiental, ya que a partir de eso pudo comprender que hay otras maneras de relacionarse con los demás seres de la naturaleza, más allá de la occidental. También hace énfasis en la importancia de la interdisciplina, el cursar materias con estudiantes y docentes de otras carreras como le ocurrió en Botánica Aplicada, Antropología General y Paisajismo, y sobre todo realizar talleres y prácticas de extensión, pues la enriqueció mucho reflexionar sobre las ideas que quiere transmitir.

Finalmente, B2 fue quien presentó la visión más diferente del resto. Si bien en un momento cuenta que gracias a cursar Botánica Aplicada y Soberanía Alimentaria pudo comprender que hay una constante interacción entre lo social y lo natural, en el resto de la entrevista presenta una postura exhabitante, donde los humanos son artificiales porque "no se recicla" lo que hacen. Cuenta que realizó numerosos voluntariados en huertas y que le sirvió mucho hablar con amigos que realizan prácticas alternativas basadas en Fukuoka [agrónomo japonés, fundador de la llamada agricultura natural (Fukuoka, 1985)]. Sin embargo, a lo largo de la entrevista presenta una visión ecocentrista.

\section{Licenciatura en Biología, Orientación Ecología}

De las entrevistadas de Ecología, dos mostraron perspectivas que se acercan a la biocentrista, mientras las restantes son claramente ecocentristas. Una de las primeras, $\mathrm{E} 2$, dice que antes se identificaba como "ecologista" ya que quería hacer algo bueno por el planeta. Sin embargo, actualmente se distancia de esa postura, ya que dice que deja afuera a las personas. Ella ve como un primer punto de inflexión, el cursar las materias sistemáticas que le mostraron la enorme diversidad que hay, lo cual le despertó entusiasmo por enseñarla y generar empatía a partir de eso. Por este motivo empezó a dar clases en un bachillerato de adultos y se hizo guía del museo. Como segundo punto, subraya el haber realizado un curso de filosofía de la ciencia, lo cual le hizo cuestionarse los saberes que le daban desde la facultad. En cuanto a la naturaleza, ella dice que todo es parte de la misma, "hasta el plástico".

Por su lado E5, presenta una postura algo más ambigua. Al momento de definir los conceptos de ambiente y naturaleza, lo hace de la manera clásica de la ecología, es decir que el primero es "todo lo que nos rodea" y la naturaleza es un sinónimo, de modo que quedan afuera "las creaciones humanas". Sin embargo, al contarme su trayectoria, desde voluntariados que realizó en la adolescencia con un enfoque conservacionista hasta su llegada a la facultad donde realizó prácticas de extensión y docencia, su perspectiva se acerca más a una biocentrista. Cuenta que experimentó un punto de quiebre fundamental durante la carrera: cursar Botánica Sistemática I con una profesora descripta como "excelente pedagoga", le hizo despertar interés por temáticas relacionadas al control de plagas, con lo que terminaría trabajando. A partir de esto empezó a investigar la historicidad del término "plaga" y de los intereses económico- 
Paolocá. Todo lo que nos rodea: estudio de representaciones sociales de lo ambiental y la naturaleza en una facultad...

políticos que esto conlleva, como el uso indiscriminado de agrotóxicos. Remarca también que la conciencia de estudiar en una Universidad laica, pública y gratuita le hizo tomar la responsabilidad de hacer algo para devolverle a la sociedad y que genere bienestar.

En los restantes casos, E1 y E3 presentan una trayectoria similar entre sí, donde ninguna realizó muchas actividades por fuera de lo estrictamente curricular, aunque E1 trabaja en una ONG ambientalista que se encarga de realizar gestión de residuos y relaciones públicas. Por otro lado, E4 si participó en numerosos proyectos de extensión en diferentes provincias, como también de viajes y pasantías con equipos interdisciplinarios, siempre dentro de las Ciencias Naturales. Sin embargo, los resultados de las entrevistas fueron muy similares, ya que las tres ven la enseñanza hacia la comunidad de manera unidireccional y pasiva ("una se olvida que la gente no sabe"), y se posicionan como exhabitantes de lo ambiental, donde las personas son intrusos que destruyen la naturaleza. E4 incluso define al ambiente como "lo que no son construcciones humanas". En lo que respecta a las falencias de la carrera, las tres coincidieron en que falta más formación en biología molecular.

\section{Licenciatura en Biología, Orientación Zoología}

En los entrevistados de zoología es donde más heterogeneidad de discursos he encontrado. Por un lado, Z3 se presenta claramente con una postura ecocentrista, donde ve a la ciudad como un lugar donde "la naturaleza está destrozada" y lo ambiental excluido; además, su crítica hacia la carrera pasa por la relación de "maltrato" de los docentes hacia los estudiantes.

Por el lado de Z1, quien arrancó la carrera por admiración hacia los "protagonistas de los documentales", su visión está más cerca del ecocentrismo que del biocentrismo, sin embargo, por su relación con lo ambiental sería más de inhabitante ya que su trabajo se basa en "contar la historia del lugar" a través de la conservación de las especies endémicas. El entrevistado remarca la contradicción que le genera conservar algo que "su destino quizás es extinguirse", pero dice que su posicionamiento es evitar que ocurra. Él nació en el campo, pero a partir de realizar viajes de campaña y pasantías en laboratorios sobre conservación, ve lo ambiental como reducido por los monocultivos.

Algo similar ocurre con Z4, aunque de forma inversa. Dice que antes veía a la naturaleza como la única que conformaba al ambiente, pero se empezó a cuestionar luego de que en su pueblo instalaran "una termo-eléctrica" que devino en conflictos socioambientales. Si bien define a los humanos como parte de lo ambiental, lo cual fortaleció a partir de realizar un ACG sobre educación ambiental y la participación en proyectos de extensión como "redescubriendo los humedales", sobre el final de la entrevista subraya que su interés es por la conservación de la naturaleza, la cual es todo aquello sin influencia humana. Si bien su perspectiva podría ser categorizada como biocentrista, su relación con el medio es de exhabitante. 
Finalmente, Z2 presenta una perspectiva más claramente biocentrista. Cuenta que antes era "más intuitiva" pero ahora es "más empírica”. Remarca como fundamental en su cambio de visión el cursar "Lógica y Epistemología de la Ciencia", a partir de la cual se empezó a cuestionar las teorías que enseñaban en la facultad como verdades inmanentes. Además, fue importante el empezar a militar en una organización de política de izquierda, donde vio cómo teorías de las Ciencias Naturales fueron utilizadas para justificar genocidios, por lo que se empezó a preguntar para qué y para quiénes se hacía ciencia. Dice que la facultad es "muy darwinista", pero los profesores hablan de la teoría sintética sin entenderla ni haber leído a Darwin. Entiende a la naturaleza y lo ambiental como construcciones teóricas.

\section{Licenciatura en Biología, Orientación en Paleontología}

De los entrevistados de Paleontología, sólo P2 mostró una visión ecocentrista. Dicho entrevistado define a la naturaleza y al ambiente como netamente biológico, dice que su pensamiento no sufrió grandes cambios a lo largo de la carrera ni participó en espacios extra académicos. Por otro lado, los restantes entrevistados mostraron una perspectiva diferente.

P1 y P4 tuvieron una trayectoria relativamente similar, no reconocen división entre lo natural y lo no natural y ambos hacen énfasis en la lectura de textos por fuera de la facultad (brindados por amigos de antropología, madre bibliotecaria y por militancia socioambiental). Critican la forma en que se enseña en la facultad, sobre todo las materias de Ecología y Evolución, y subrayan la importancia de las salidas de campo y los viajes en su formación. Ambos dicen que la materia Lógica fue la más importante en la conformación de su pensamiento. Remarcan que a la formación le hace falta más filosofía y que se hable de la noción política del científico.

En lo que respecta a P3, si bien su postura no se distancia demasiado de los anteriores, su trayectoria fue algo diferente. Participó en varios proyectos de extensión y trabaja en la importancia del patrimonio para la conformación de identidades. Entiende lo ambiental como social y biológico.

\section{Licenciatura en Geología}

En geología, G3 y G4 definieron a lo ambiental desde un enfoque social y biológico, haciendo énfasis en las interrelaciones. Ambas notaron que, antes de empezar la facultad, pensaban que el ambiente solo se circunscribía a las plantas y los animales, sin embargo, no fue la carrera en ninguno de los dos casos lo que las hizo cambiar. Al menos no las materias ni los profesores de manera directa, sino que, por un lado G3 cuenta que fue un punto de inflexión su militancia en una agrupación estudiantil de centro-izquierda, a partir de la cual empezó a ver a los recursos naturales como "bienes comunes". En el caso de G4, fue cierta línea que sentía que bajaba desde el discurso de los profesores de las materias específicas de geología lo que le empezó a hacer ruido, por lo que decidió investigar por su cuenta y leer otras disciplinas como la historia. A su 
Paolocá. Todo lo que nos rodea: estudio de representaciones sociales de lo ambiental y la naturaleza en una facultad...

vez, dice que fue importante conocer gente de zonas mineras que desde la facultad se las definía como muy beneficiadas por dicha práctica, pero que sin embargo vivían en condiciones mucho peores que antes del inicio de la explotación del yacimiento minero. En los dos casos, son muy críticas de la formación de la facultad, ya que la misma tendría un lineamiento antropocentrista. En esta última línea se encontraría G1, quién remarca su "pasión por la geología" y dice que gracias a su formación en la carrera ahora entiende mucho mejor los procesos del planeta. Al hablar del rol profesional, contó la importancia de conocer los procesos del interior y la superficie de la tierra en las prácticas extractivistas. Su postura respecto a lo ambiental es claramente de exhabitante; ve a la naturaleza como todo lo que no es humano.

Finalmente, G2 y G5, se posicionan desde un ecocentrismo, puesto que dicen sentirse ambientalistas, incluso una de ellas participa en Greenpeace. Sin embargo, presentan una marcada visión dualista de la naturaleza, e incluso sus concepciones de ambiente son estrictamente geológicas. Al igual que G1, no participaron de espacios externos a lo estrictamente académico, ni presentaron cambios en su visión durante la carrera. Coincidieron en remarcar como importantes los conocimientos que les brindó la facultad, y si bien ambas separaron a los humanos de la naturaleza, G5 enfatizó a "lo verde" como lo más natural.

\section{Licenciatura en Antropología}

En antropología, dentro de sus diferencias, los cinco entrevistados presentaron una visión más ligada al biocentrismo. En cuanto a la naturaleza, fue llamativa la coincidencia de que fue a partir de la carrera que comenzaron a tener una visión no dualista de la misma ya que antes pensaban a los humanos fuera de ella y, a su vez, no formaban parte de lo ambiental.

A1 y A2 presentaron una trayectoria similar, pues ambas vinieron del campo y presentaron desde la escuela una preocupación por las fumigaciones y usos de agrotóxicos. A1 es hija de padres que militan en una "organización ecologista", por lo que dice que fue fundamental en su formación, la participación desde chica en marchas y asambleas. A su vez, dice que durante la carrera pudo empezar a comprender "lo simbólico y económico de lo ambiental" y que ahora piensa que somos las personas quienes hacemos al ambiente y que su estudio sirve, por otro lado, para entendernos a nosotros mismos. De la misma manera, el realizar obras de teatro y talleres de extensión con dicho enfoque, le sirvió para seguirse formando y poder comprender mejor cómo la gente se relaciona con lo ambiental. En cuanto a A2, ella enfatizó la importancia de cursar las materias arqueológicas tales como Arqueología Americana I, puesto que le mostraron diferentes formas de relación entre los humanos con los otros seres vivos a lo largo de la historia. También tuvo críticas para con la formación de la facultad, ya que dijo que es "demasiado naturalista" y debería tener un enfoque más hacia la gestión.

En esta última línea coincide con A5, ya que ella remarcó las mismas falencias y le sumó la falta de conocimiento de historia dentro de lo impartido por la facultad, lo cual ella lo suplió cursando optativas. Dijo que hubo dos puntos de quiebre fundamentales 
para llegar a conformar su conceptualización actual de lo ambiental: uno fue el realizar la Actividad Complementaria e Grado (ACG) de Educación Ambiental de la FCNyM y dar, en el marco de la misma, talleres en las escuelas. El otro fue trabajar con chicos de barrios populares en "Casa Joven", lo que le hizo ver la faceta política del ambiente. De esta manera remarcó que lo ambiental se caracteriza fundamentalmente por las "emergencias propias que se generan" a partir de las interacciones entre las sociedades humanas y los restantes seres vivos. Su postura es marcadamente inhabitante.

A3, por su lado, decidió anotarse en antropología porque quería estudiar a los humanos "en relación con las cosas del mundo". Cuenta que fue muy importante para comprender mejor esta relación, el leer a filósofos latinoamericanos donde mostraban una relación particular con el entorno que era distinta a la de otros pensadores. De esta manera rompió con su visión "ecologista" de ver a la naturaleza como estática. Cuenta que también fue importante realizar prácticas pedagógicas, ya que empezó a ver a la ciencia como abierta a las personas y a conocer mejor sus realidades. Es en esta diversidad de pensamientos y enfoques que empezó a romper con el dualismo sociedades-ambiente. En esta misma línea se posiciona A4, quien remarcó que antes tenía un "enfoque ecologista", pero que a partir de cursar materias como Antropología Sociocultural I y II, se dio cuenta que era el posicionamiento teórico lo que conformaba lo ambiental y construía las categorías.

\section{Discusión y conclusiones}

A partir de la información recolectada, hay algunas conclusiones que saltan a primera vista. Desde las carreras de la FCNyM se enseña lo ambiental y a la naturaleza desde un planteo dualista, al cual se le puede aplicar el concepto de hegemonía en términos de Antonio Gramsci (Thwaites Rey, 1994), es decir como serie de mecanismos de transmisión ideológica tendientes a lograr un consenso que le otorga bases más sólidas a la dominación.

De esta manera, el concepto hegemónico de ambiente dentro del ámbito de la FCNyM es "todo lo que nos rodea", el cual es repetido por la mayoría de los entrevistados, pero no es apropiado por ellos de manera homogénea. Por otro lado, hubo una coincidencia total con respecto a la conceptualización que tenían del mismo antes de empezar la facultad. El ambiente se encontraba restringido a los seres vivos no humanos y los factores abióticos, oponiéndose al mundo de las personas. Dicha reflexión nos muestra cuál es el concepto hegemónico de ambiente, y a su vez, que se percibe una dicotomía entre naturaleza y seres humanos.

Si bien prácticamente todos los entrevistados se refieren al transcurso dentro de la facultad como un gran cambio, en algunos casos esto se corresponde con un aumento de conocimiento teórico específico de la disciplina, y no necesariamente con un cambio de perspectiva con respecto a lo ambiental. Dentro de quienes presentaron un viraje hacia una perspectiva biocentrista, los factores determinantes de este cambio fueron, ante todo, el cuestionamiento de cierto discurso impartido por docentes de la facultad que se presentaba como objetivo y único. De esta manera, se acercaron a disciplinas 
Paolocá. Todo lo que nos rodea: estudio de representaciones sociales de lo ambiental y la naturaleza en una facultad...

tales como la historia, la filosofía y la lógica, ya sea de forma autodidacta, por cursos extracurriculares o mediante el cursado de materias optativas y/u obligatorias. Dentro de estas últimas sobresalió la presencia de "Lógica y Epistemología de las Ciencias", "Antropología General" y "Botánica Aplicada" entre los biólogos, y "Antropología Sociocultural I", "Antropología Sociocultural II" y "Arqueología Americana I" entre los antropólogos (FCNyM, s/f a). No hubo materias destacadas entre los geólogos. Al analizar dichas asignaturas se observó, como punto en común, el presentar una perspectiva interdisciplinaria, desde la conformación del plantel docente hasta en el desarrollo del contenido. Por otro lado, al estudiar el marco de producción del conocimiento científico, se deja de percibir al mismo como inmutable y permite el cuestionamiento del saber hegemónico. Por último, y en relación con el punto anterior, todas estas materias coinciden en darle cierta profundidad histórica a su análisis, y contraponen diversas escuelas de pensamiento, lo que aporta a una concepción dinámica y política -en términos amplios- de la ciencia. Es por ello que contribuyen a romper con el antropocentrismo, y particularmente, con el ecocentrismo dualista.

En lo que respecta a las ACG, fue repetidamente nombrada la de Educación Ambiental de la FCNyM (FCNyM, s/f b), la cual cuenta dentro de su plantel docente con egresados y estudiantes de diferentes carreras de la facultad. Varios de los entrevistados también hicieron énfasis a la apertura teórica que les generó empezar a militar en organizaciones políticas de izquierda y centro-izquierda, al acercarse a lecturas críticas de teorías de las Ciencias Naturales y su utilización para justificar prácticas sociales antropocentristas, así como también al papel que cumplieron en el pasado como legitimadoras de genocidios. Dentro de este grupo de entrevistados, los mayores reclamos hacia el plan de estudios de sus respectivas carrera, se centraron en la falta de formación en historia, filosofía y en gestión. Mientras quienes presentaron una perspectiva diferente, no hicieron comentarios con respecto a estos campos, sino más bien enfocados a contenidos tales como la falta de formación en microbiología y, en menor medida, mastozoología. En lo que respecta exclusivamente a los entrevistados de antropología, fue muy criticado el enfoque naturalista de la carrera, sin embargo, en otros tramos de la entrevista resaltaban la importancia de estudiar a las personas insertadas en lo ambiental y en relación con los demás seres vivos. Por último, dentro de geología, quienes fueron críticos de la formación, hicieron énfasis en la línea extractivista de las cátedras, las cuales tendrían una fuerte perspectiva antropocentrista.

Por otro lado, la mayor heterogeneidad de discursos se encontró entre los entrevistados de biología con orientación en zoología, siendo una posible explicación a este fenómeno las diferentes trayectorias seguidas por los entrevistados. Mientras que la entrevistada que aún era estudiante, y concurría a la facultad sólo para cursar poseía una visión más ligada a la hegemónica, la militancia política y la formación en filosofía de la biología de Z2 resultó clave en la adopción de una perspectiva más crítica. Los restantes dos casos de zoología fueron de los más difíciles de analizar al presentar cierta ambivalencia en sus discursos. Es posible que esto se deba a un momento en sus 
trayectorias, donde se encuentran cuestionándose ciertas ideas previas o redefiniéndolas en base a la oposición con las de otros.

En lo que respecta a las prácticas docentes y de extensión, las mismas no son determinantes por sí mismas en la conformación de representaciones, sino que es el enfoque de las mismas y la manera de interacción con los destinatarios lo que resulta fundamental. Aquellos que perciben a ambas prácticas de una manera bancaria -es decir, "que el único margen de acción que se ofrece a los educandos es el recibir los depósitos, guardarlos y archivarlos (...) [lo cual] sólo les permite ser coleccionistas o fichadores de cosas que archivan" (Freire, 1972, p. 39)- resultaron ser quienes mantenían una visión dualista y ecocentrista. Por otro lado, quienes mostraron un enfoque biocentrista, dicen haber generado relaciones de empatía con los destinatarios y haber reflexionado $\mathrm{y}$ aprendido a partir del encuentro con ellos y, fundamentalmente, con el territorio. En varios de estos casos, contaron que al poder conocer más profundamente el mundo de quienes acudían a los talleres les hizo cuestionarse su propia posición con respecto a qué es lo ambiental, incorporando valoraciones sociales, políticas y económicas. Dichas reflexiones concuerdan con Vessuri (2008), quién dice que la ciencia tiene una relación de interdependencia y retroalimentación con la política, y por ende, los conocimientos de la primera son producto de procesos de construcción social de carácter participativo y transdisciplinario -éste último concepto entendido en términos de Lawrence y Després (2004)- es decir, incorporando conocimientos de profesiones por fuera de la investigación científica tradicional.

En la misma sintonía, las prácticas interdisciplinarias sirvieron para complejizar la visión de los entrevistados sólo en el caso de presentarse una interacción con disciplinas de otras ramas u oficios, distintos a los estrictamente biológicos. Aquellos que decían pertenecer a equipos interdisciplinarios donde sólo había gente de diferentes ramas de la biología, su perspectiva se mantuvo dualista. Es por eso que una práctica interdisciplinaria resulta determinante al conjugar profesionales de las Ciencias Naturales y de las Sociales. Es por ello que el concepto de biocentrismo, propuesto por Gudynas (2010, p. 51) resulta superador para una comprensión holística, crítica y comprometida de lo ambiental y la naturaleza; así, "alerta que existen muchos otros valores de origen humano [además del económico], tales como aquellos que son estéticos, religiosos, culturales, etc., les suma valores ecológicos (...), e incorpora los valores intrínsecos". De esta manera, busca superar la visión de naturaleza como un objeto, al abandonar el dualismo antropocentrista.

Finalmente, aquellos que presentaron una perspectiva ecocentrista, se mostraron a su vez como exhabitantes, los cuales afectan al "ambiente" pero no se ven influidos por él. Se observan a sí mismos como externos de lo ambiental, y nombran a las personas y a sus "construcciones" como opuestas a la naturaleza, pese a ello, ven como fundamental su intervención para evitar que las personas la destruyan. Por lo tanto, no solo se estarían viendo como habitantes externos del mundo, sino también de lo social.

De esta manera, se revela como determinante en la deconstrucción de un enfoque dualista de la naturaleza y de lo ambiental, un replanteamiento del rol del 
Paolocá. Todo lo que nos rodea: estudio de representaciones sociales de lo ambiental y la naturaleza en una facultad...

profesional, es decir de la ciencia, por qué y para quiénes (Mengascini et al., 2004), y un cuestionamiento del conocimiento como único y objetivo. Se ha observado cómo una práctica interdisciplinaria, que conjugue ciencias sociales y naturales, es fundamental para un enfoque verdaderamente biocentrista. En este sentido, las entrevistas reflejan cómo a partir de la desnaturalización e historización de los conceptos hegemónicos imperantes en la biología, se produce un cambio obligatorio de perspectiva que ayuda a romper el dualismo ambiente-sociedad y naturaleza-humano. Al igual que en la contribución de Cordero et al. (2011), el presente trabajo arroja como resultado, la importancia de lo que estas autoras llaman investigación-acción participativa en la configuración del desarrollo del perfil profesional de investigadores y docentes universitarios, las autoras remarcan el carácter colectivo, interdisciplinario y político de dicha práctica.

Se estima que ése es uno de los motivos por el cual dentro de las carreras de Ecología y Geología, las cuales no presentan materias con contenido social en su currícula, se han encontrado la mayor cantidad de posturas ecocentristas. En sintonía, vemos como importante la presencia de la carrera de Antropología en una facultad de Ciencias Naturales, ya que ayuda a sus estudiantes a conformar una concepción más holística de lo ambiental, y, a su vez, aporta a la formación de otras disciplinas como es el caso de Botánica, cuyos estudiantes remarcaron como materias claves a Antropología General y Botánica Aplicada, las cuales cuentan con un enfoque antropológico.

En lo que respecta a lo que no es estrictamente curricular, surge como importante la realización de una práctica de extensión y de docencia con un enfoque crítico, interdisciplinario y de compromiso territorial. Dichas prácticas, al entablar una relación de empatía e interpelación con la comunidad, resulta en una formación de mutuo aprendizaje. Sin embargo, si se realizan de manera bancaria (Freire, 1972), donde el profesional va a depositar el conocimiento en el destinatario, sin importarle el contexto socio-político del mismo, no contribuirá a una visión no dualista. Es por eso que es imperante poner en discusión el paradigma institucional tradicional de la educación superior, y remplazarlo por una perspectiva de aprendizaje-servicio (Tapia, 2008). Desde este enfoque la Universidad se reconoce parte de la comunidad, sin subordinarse a demandas del mercado, entablándose una relación dialéctica y dinámica entre aprendizaje, investigación e intervención social. Así, "al involucrarse en la resolución de problemáticas reales de una comunidad específica, la Universidad trabaja con realidades complejas, que se resisten a ser abordadas sólo desde la mirada estrecha de una disciplina académica, y por eso los proyectos de aprendizaje-servicio tarde o temprano se ven obligados a superar los compartimentos estancos (...) de las disciplinas hiperespecializadas, y se abren a la interdisciplina, a la multidisciplina y a las disciplinas 'híbridas"' (Tapia, 2008, p.16).

Este trabajo tuvo por finalidad ser una aproximación al estudio derepresentaciones sociales del ambiente y la naturaleza de estudiantes avanzados y graduados recientes de la FCNyM, debido a la importancia que tiene formar profesionales capacitados para actuar sobre las problemáticas socioambientales contemporáneas. En consonancia, al ser la UNLP una Universidad pública y gratuita, es fundamental la promoción de sujetos 
comprometidos y con conciencia social que generen conocimientos en pos del bienestar humano y de los seres vivos en general. Espero que este trabajo sea un aporte para la discusión actual en torno a la renovación de los planes de estudios de las licenciaturas de la FCNyM, y que de esta manera las problemáticas socioambientales sean un eje transversal a la hora de desarrollar los mismos. La formación de sujetos conscientes y críticos es la única manera de superar la crisis ambiental actual.

\section{Agradecimientos}

Agradezco a las entrevistadas y entrevistados por su inestimable colaboración con esta investigación, así como su autorización para difundir los resultados; a "Tany" Pochettino (LEBA, FCNyM-UNLP), Aylén Capparelli (FCNyM, UNLP) y revisores anónimos por las sugerencias que aportaron a mejorar este trabajo; a Ignacio Requena (SBCC, EE.UU.) por la revisión del inglés. Este estudio lo realicé en el marco de una Beca de Estímulo a las Vocaciones Científicas - Consejo Interuniversitario Nacional (EVC-CIN).

\section{Conflicto de intereses}

Declaro que no hay conflicto de intereses que puedan haber influido en los resultados obtenidos o en las interpretaciones propuestas.

\section{Referencias bibliográficas}

Boas, F. (1964) Cuestiones Fundamentales de Antropología Cultural. Buenos Aires, Argentina: Solar Hachette.

Cordero, S.; Dumrauf, A.G.; Mengascini, A. y Sanmartino, M. (2011) Entre la Didáctica de las Ciencias Naturales y la Educación Popular en Ciencias Naturales, Ambiente y Salud: relatos y reflexiones de un camino en construcción. Praxis Educativa, 15 (15), pp. 71-79.

Da Silva, T.C.; Cruz, M.P.; De Sousa Araújo, T.A.; Schwarz, M.L. y Albuquerque, U.P. (2014) Chapter 7: Methods in Research of Environmental Perception. En Albuquerque, U.P; Fernandes Cruz Da Cunha, L. V; Paiva De Lucena, R. F. y Nóbrega Alves, R. R (Comps.), Methods and Techniques in Ethnobiology and Ethnoecology. New York: Humana Press, pp. 99-109.

Descola, P. y Pálsson, G. (2001) Introducción. En Descola, P. y Pálsson, G. (Comps.) Naturaleza y sociedad: perspectivas antropológicas. México D.F.: Siglo XXI, pp. 11-33.

Durand, L. (2002) La relación ambiente-cultura en antropología: Recuento y perspectivas. Nueva Antropología, 18(61), pp. 169-184.

Eckersley, R. (1992) Environmentalism and Political Theory. London, United Kingdom: University College London. 
Paolocá. Todo lo que nos rodea: estudio de representaciones sociales de lo ambiental y la naturaleza en una facultad...

FCNyM (Facultad de Ciencias Naturales y Museo) - UNLP(s/fa). Cátedras. Recuperado de: https://www.fcnym.unlp.edu.ar/_catedras

FCNyM(Facultad de Ciencias Naturales y Museo)-UNLP(s/fb). ACG Educación Ambiental. Recuperado de: https://www.fcnym.unlp.edu.ar/acg_educacion_ambiental

Fowler, C. (1979) Etnoecología. En Hardesty, D. Antropología Ecológica. Barcelona: Bellaterra, pp. 215-238.

Freire, P. (1972) Pedagogía del Oprimido. Buenos Aires, Argentina: Siglo XXI.

Fukuoka, M. (1985) The Natural Way Of Farming: The Theory and Practice of Green Philosophy. New York, U.S.A.: Japan Publications.

García, D. (2009) Educación ambiental: Aportes políticos y pedagógicos en la construcción del campo de la Educación Ambiental. Buenos Aires, Argentina: Secretaría de Ambiente y Desarrollo Sustentable de la Nación.

Gudynas, E. (1999) Concepciones de la naturaleza y desarrollo en América Latina. Persona y Sociedad, 13(1), pp. 101-125.

Gudynas, E. (2004) Ecología, economía y ética del desarrollo sostenible. Montevideo, Uruguay: Coscoroba.

Gudynas, E. (2010) La senda biocéntrica: valores intrínsecos, derechos de la naturaleza y justicia ecológica. Tabula rasa, ${ }^{\circ} 13$, pp. 45-71.

Hunn, E. (2007) Ethnobiology in four phases. Journal of Ethnobiology, 27(1), pp. 1-10. Ingold, T. (2012) Ambientes para la vida. Conversaciones sobre humanidad, conocimiento y antropología. Montevideo, Uruguay: Trilce.

International Society of Ethnobiology. (2008) Code of Ethics, Recuperado de: http://www. ethnobiology.net/what-we-do/core-programs/ise-ethics-program/code-of-ethics/

Jodelet, D. (1984). La representación social: fenómenos, concepto y teoría. En Moscovici, S. (Comp.), Psicología Social II. Barcelona, España: Paidós, pp. 469-443.

Lawrence, R. y Després, C. Futures of Transdisciplinarity. Futures, 36, 2004, pp. 397-405. Leopold, A. (1949) A Sand County Almanac. And Sketches Here and There. Oxford, United Kingdom: Oxford University Press.

Martin, G. (1995) Ethnobotany, A methods manual. London, U.K.: People and Plants International Conservation.

Mastrángelo, A.V. (2009) Análisis del concepto de recursos naturales en dos estudios de caso en Argentina. Ambiente \& Sociedade, 12(2), pp. 341-355.

Mastrángelo, A.V. (2015) Derechos de humanos y no humanos: una reflexión basada en dos estudios de caso etnográficos. Caderno Eletrônico de Ciências Sociais, 3(1), pp. 35-53.

Mengascini, A.; Menegaz, A.; Ros, M.; Arnedillo, M.P.; Sarutti, A. y Ottenheimer, A.C. (2003) Revisión curricular en la Facultad de Ciencias Naturales y Museo, UNLP: recorrido de los estudiantes y valoración de espacios formativos no obligatorios. Actas 2-10, Congreso Latinoamericano de Educación Superior en el Siglo XXI. San Luis, Argentina.

Mengascini, A.; Menegaz, A.; Murriello, S. y Petrucci, D. (2004) Yo así, locos como los vi a ustedes, no me lo imaginaba: Las imágenes de ciencia y de científico de estudiantes de carreras científicas. Enseñanza de las Ciencias, 22(1), pp. 65-78. 
Milton, K. (1997) Ecologías: antropología, cultura y entorno. Revista Internacional de Ciencias Sociales, 154, pp. 86-115.

Ortner, S.B. (1984) Theory in Anthropology since the Sixties. Comparative Studies in Society and History, 26(1), pp. 126-166.

Ottenheimer, A.C.; Menegaz, A.; Mengascini, A.; García, D.; Gardinetti, A.; Arnedillo, M.P. y Bover, T. (2004) Aproximación a la formación para la investigación científica en Ciencias Naturales. Actas 1-10, VII Congreso Argentina de Antropología Social. Villa Giardino, Argentina.

Society of Ethnobiology (s/f). Recuperado de: https://ethnobiology.org/

Tapia, N. (2008) Aprendizaje y servicio solidario en la misión de la Educación Superior. En Tedesco, J. C. y Tapia, N. (Comps.). El aprendizaje- servicio en la Educación Superior. Una mirada analítica desde los protagonistas. Buenos Aires, Argentina: EUDEBA, pp. 11-34.

Thwaites Rey, M. (1994) La noción gramsciana de hegemonía en el convulsionado fin de siglo. EN L. Ferreyra, L; Logiudice, E. y Thwaites, R. M (Comps.). Gramsci mirando al sur: Sobre la hegemonía en los 90. Buenos Aires, Argentina: Kohen y Asociados Internacional, 1994, pp. 15-84.

Vessuri, H. (2008) Capítulo 2: El futuro nos alcanza: mutaciones previsibles de la ciencia y la tecnología. En Gazzola, A. L y Didriksson, A. (Comps.), Tendencias de la Educación Superior en América Latina y el Caribe. Caracas: IESALC-UNESCO, pp. 55-86.

Wyndham, F.S.; Lepofsky, D. y Tiffany, S. (2011). Taking stock in ethnobiology: Where do we come from? What are we going? Journal of Ethnobiology, 31, pp. 110-127. 


\section{Anexo 1 - Modelo de entrevista}

1. ¿Qué carrera estudiás o estudiaste y en qué año ingresaste a la facultad?

2. ¿Por qué elegiste esa carrera?

3. ¿Qué entendés por ambiente?

4. ¿Qué relación ves entre tu carrera y lo ambiental?

5. ¿Qué es la naturaleza?

6. ¿Te acordás qué pensabas de estos conceptos antes de ingresar?

7. ¿Tu idea de ambiente y/o naturaleza fue cambiando a lo largo de la carrera?

8. (En caso de respuesta afirmativa) ¿Hubo algún punto de quiebre o algún factor importante que influyó en este cambio de pensamiento?

9. ¿Qué materias optativas hiciste?

10. ¿Sentís que la temática ambiental se relaciona con algún otro espacio de tu vida?

11. ¿Hay algo más que te resulte relevante contarme? 


\section{Anexo 2 - Modelo de solicitud de consentimiento (previamente informado) de participación en la investigación}

La Plata, (fecha)

Estimada/o participante:

Gracias por destinar su tiempo para participar y dar el consentimiento para la realización del presente trabajo de beca. El propósito de este trabajo, es conocer un poco más sobre la trayectoria y la formación durante el transcurso de la carrera, en la zona de La Plata y cercanías en el marco de un trabajo de la investigación.

Los resultados podrán ser útiles para la comunidad universitaria en particular y la sociedad en su conjunto.

Reconozco que no obtendré un beneficio directo porparticipar de la presente investigación, pero que mi aporte podrá genera conocimientos para las futuras generaciones.

Si está de acuerdo con la propuesta, por medio de esta carta declaro mi voluntad de permitir la difusión:

Mi nombre y apellido si no

Grabaciones si no $\square$

Conocimientos si no

Acepto participar de la entrevistas realizadas por Iván Paolocá en el marco de su trabajo de investigación, siempre y cuando se me reconozca como fuente de información.

Una vez más, agradezco mucho su cooperación.

Firma

Aclaración 
